\title{
Auxiliary diagnostic potential of ventricle geometry and late gadolinium enhancement in left ventricular non- compaction; non-randomized case control study
}

\author{
Marko Boban ${ }^{1,2^{*}}$ D, Vladimir Pesa', Ivo Darko Gabric ${ }^{3}$, Sime Manola ${ }^{3}$, Viktor Persic ${ }^{1,2}$, Helena Antic-Kauzlaric ${ }^{1}$, \\ Marinko Zuljj and Aleksandar Vcev ${ }^{2}$
}

\begin{abstract}
Background: There are still ambiguities existing in regard to left ventricular non-compaction (LVNC) diagnostic imaging. The aim of our study was to analyze diagnostic potential of late gadolinium enhancement (LGE) and ventricle geometry in patients with LVNC and controls.

Methods: Data on cardiac magnetic resonance imaging (CMR) studies for LVNC were reassessed from the hospital's database (3.75 years; $n=1975$ exams). Matching sample of controls included cases with no structural heart disease, hypertrophic or dilative cardiomyopathy, arrhythmogenic right ventricular dysplasia or subacute myocarditis. Eccentricity of the left ventricle was measured at end diastole in the region with pronounced NC and maximal to minimal ratio (MaxMinEDDR) was calculated.
\end{abstract}

Results: Study included 255 patients referred for CMR, 100 (39.2\%) with LVNC (prevalence in the studied period 5.01\%) and 155 (60.8\%) controls. Existing LGE had sensitivity of 52.5\% (95\%-Cl:42.3-62.5), specificity of 80.4\% (95\%-Cl:73.2-86.5) for LVNC, area under curve (AUC) 0.664 (95\%-Cl:0.603-0.722); $<<0.001$. MaxMinEDDR>1.10 had sensitivity of 95.0\% (95\%Cl:88.7-98.4), specificity of 82.6\% (95\%-Cl: 75.7-88.2) for LVNC, AUC 0.917 (95\%-Cl:0.876-0.948); $p<0.001$. LGE correlated with Max-Min-EDD-R (Rho=0.130; $p=0.038$ ) and there was significant difference in ROC analysis $\triangle \mathrm{AUC0.244} \mathrm{(95 \% -Cl:0.}$ 175-0.314); $p<0.001$. LGE also correlated negatively with stroke volume and systolic function (both $p<0.05$, respectively).

Conclusions: LGE was found to be frequently expressed in patients with LVNC, but without sufficient power to be used as a discriminative diagnostic parameter. Both LGE and eccentricity of the left ventricle were found to be relatively solid diagnostic landmarks of complex infrastructural and functional changes within the failing heart.

Keywords: Left ventricle non-compaction (LVNC), Late gadolinium enhancement (LGE), Cardiac magnetic resonance imaging (CMR), Left ventricular geometry

\footnotetext{
* Correspondence: marcoboban@yahoo.com

'Department of Cardiology, University hospital "Thalassotherapia Opatija",

Faculty of Medicine, University of Rijeka, M. Tita 188/1, 51440 Opatija, Croatia

2Department of Internal medicine, Faculty of Medicine, "J.J. Strossmayer"

University of Osijek, Osijek, Croatia

Full list of author information is available at the end of the article
} 


\section{Background}

Left ventricle non-compaction (LVNC) is cardiomyopathy of infrequent prevalence, but associated with serious adverse prognostic outcomes [1,2]. There is a complex pathophysiologic background made up of inherited and external factors, whose interplay leads to alterations in cellular organization or signaling, myocardial wall maturation, loss of systolic function, increased likelihood of arrhythmias and intracardial thrombosis [3-5]. On the other side, due to great variability of clinical expression and presentation, currently available diagnostic criteria are not perfect [6]. Trabeculations of the left ventricle can indeed be found in non-negligible number of imaging studies, but their clinical significance is often inconclusive, particularly in cases in which prognostic assessment is solely based on imaging. In some cases genetic testing can offer valuable landmark that can reveal a concealed risk of sudden cardiac death $[7,8]$.

Specific characteristics of LVNC, such as compact (C) myocardial wall thinning, pronounced meshwork of non-compact $(\mathrm{NC})$ myocardium with 2.0-2.3 ratio of $\mathrm{NC} / \mathrm{C}$ and a proof of blood flow through the trabecula, taken cumulatively together make that only some cases with trabeculated myocardium fit within the LVNC cardiomyopathy [9-11]. Proportion of non-compact myocardium in the total mass of the left ventricle was also found to be a highly sensitive and specific diagnostic parameter in cases in which over one fifth of the ventricle is affected [12]. Remarkable advancements in diagnosing LVNC came with wider availability of cardiac magnetic resonance imaging (CMR) $[13,14]$. Thanks to excellent insight in volumetrics, three dimensional multiplanarity, geometry and tissue changes, CMR offers valuable data for verification of the LVNC diagnosis, as well as functional analysis and insight in the prognostic course, superior to the conventional imaging modalities $[12,15]$. Tissue characterization, especially the late gadolinium enhancement (LGE), offer further information on pathophysiology, differential diagnosis, as well as pathoanatomic background for increased prevalence of arrhythmias or thromboses [16, 17].

Previous studies have shown that existence of LGE in LVNC is associated with deprived prognostic course $[18,19]$. Left ventricle (LV) geometry was only scarcely studied in regard to LVNC [17]. Our preliminary findings, based on large volume of exams, found that eccentricity of the left ventricle is connected with LVNC. The aim of our study was to assess the existence, characteristics and diagnostic potential of LGE in patients with LVNC and controls, using CMR. In addition, connections existing between LGE and geometry of the left ventricle were systematically assessed, with particular interest on their head to head diagnostic utility.

\section{Methods}

Patients referred for CMR imaging of non-ischemic cardiomyopathies were prospectively included in our registry after signing an informed consent, prior to imaging procedure. Study was conducted in accordance with the Declaration of Helsinki and good clinical practice principles. Approval was obtained from the ethical board of the Hospital. There was no funding, compensations or other sources of financing, and there was no relation with medical industry. There were no additional or indirect reimbursements for patients or study personnel.

Patients included in this study were retrospectively recruited from a digital CMR data base (including analyses for diagnostic reports and image files), based on LVNC diagnosis, for a period of 3.75 years. Entire set of imaging studies was reanalyzed by two experienced cardiologists and a radiologist. LVNC diagnosis included the following CMR diagnostic criteria: non-compact-tocompact layer $>2.3: 1$, thinning of the compact myocardium layer and trabeculations affecting $>20 \%$ of the left ventricle $[3,10,12]$. There were $2(0.1 \%)$ cases with clinically confirmed LVNC not fulfilling the criterion of $\mathrm{NC}>20 \% \mathrm{LV}$. In those cases, LVNC diagnosis was confirmed with the following clinical criteria: electrocardiographic abnormalities, electrophysiology testing and in one case, family history of cardiomyopathy and sudden cardiac death. Cases with existing trabeculations whose range did not fulfill the previously mentioned CMR criteria in a clearly defined manner were included as a subgroup of controls i.e. as the cases with suspected LVNC. This sub-group of controls included patients with $\mathrm{NC} / \mathrm{C}$ ratio range of $1.8-2.2: 1$ and trabeculations affecting $>10 \%$ to $<17 \%$ of LV. Control group of patients included statistically comparable sample of individuals with: no evidence of structural heart disease (healthy athletes, those with arrhythmias and those referred to CMR for ruling out of structural heart disease, prior to electrophysiology studies, cardiac thrombosis or cardiomyopathy), hypertensive heart disease (HHD), hypertrophic cardiomyopathy (HCM), dilative cardiomyopathy (DCM) (considering exclusion of significant coronary artery disease), arrhythmogenic right ventricle cardiomyopathy (ARVD) and subacute or late phase myocarditis (inclusion of outpatients). Study did not include patients with congenital heart disease, previous heart surgery, cardiac tumors, significant pericardial effusion, primary valvular disorder (aortic stenosis, mitral stenosis) and those with coronary artery disease (having significant coronary artery disease (known stenoses $>30 \%$ ), positive adenosine stress test for screening of coronary artery disease, or ischemic type of late gadolinium enhancement).

Imaging studies were performed on $1.5 \mathrm{~T}$ Magnetom Avanto, Siemens $^{\oplus}$ (Erlangen, Germany, EU), using ECG gating and breath-hold following two respiratory cycles, 
Body Matrix chest and spine coils. CMR protocol consisted of setting localizers, Half-Fourier Acquisition Single-shot Turbo spin Echo (HASTE) sequences, steady state free precession (SSFP) of standard heart 2-, 4- and 3 -chamber planes and $6 \mathrm{~mm}$ stack of short axial slices (8-12 slices through ventricle), with adding of the right ventricle and its outflow tract in case of any clinical questions. In certain cases, that was followed by short tau inversion recovery (STIR) or turbo spin echo (TSE) $\mathrm{T} 1$ and $\mathrm{T} 2$ sequences dark blood, and fat saturation sequences, which was indicated prior to or during the exam, on a case-to-case basis. Gadolinium contrast was applied in a $0.2 \mathrm{~mL} / \mathrm{kg}(0.1 \mathrm{mmol} / \mathrm{kg})$ dose. An intravenous bolus of Omniscan ${ }^{\circ}$ [Gadodiamide, GE Healthcare, Little Chalfont, UK, EU] or Dotarem ${ }^{\circ}$ [gadoterate meglumine, Guerbet, Roissy, France, EU] was used in the exams, as well as late gadolinium enhancement (LGE) sequences 20-30 min following the contrast application.

Postprocessing was performed on Siemens AGNUMARIS/4, Syngo MR B17 ${ }^{\circ}$ software package (Erlangen, Germany, EU), whilst volumetric analyses were done using Siemens AG- Syngo Console Argus ${ }^{\circ}$, by a team made of two CMR high throughput cardiologists (300-450 exams per year) and a radiologist. Standard reports included a clinical questionnaire from the referring cardiologist, medical history, interpretation of all planes, sequences, tissue sequences, volumetrics, dedicated measurements of myocardial thickness in trabecula and solid part, indirect analysis of valvular function, late gadolinium enhancement and conclusions arrived at based on the exam. Final interpretation of the results was done in accordance with the standardized myocardial segmentation, as recommended by professional associations. For the purpose of this study, geometry included eccentricity analyses in the dedicated region of the left ventricle, affected by non-compaction, and the same i.e. comparable region in the control group of patients, developed by the principal author of the study (MB). Slices from the mid to apical part (chosen from the 4th - 8th layer of short axis stack of $6 \mathrm{~mm}$ slices, with typical range 8-12 per patient) of the left ventricle short axis single slice, created parallel to mitral valve by calculating the ratio of the longest end diastolic diameter (EDD) divided by the diameter of perpendicular line (solid to solid myocardium), i.e. maximal/minimal end diastolic ratio (MaxMinEDDR).

Population and studied groups were analyzed using descriptive statistic and presented as means combined with standard deviations or numbers with percentages. Numeric variables were analyzed for differences by the Mann-Whitney U test or Kruskal Wallis. Group data analyses were calculated with Chi square or Kruskal Wallis. Connections between the studied CMR parameters and LVNC were described by Spearman Rho.
Bi-nominal regression analysis was used for the studied CMR parameters and LGE existence. LVNC diagnostic value of the late gadolinium enhancement and left ventricular geometry was first calculated separately and then in head to head settings using receiver operating characteristic (ROC) curve analysis and NC/C plus NC $>20 \%$ LV as previously standardized parameters. $P$ value less than 0.05 was considered significant. Statistical analyses were done by an experienced statistician using IBM$\mathrm{SPSS}^{\circ}{ }^{\circ}$ v 20 (IBM co, Chicago, IL, USA) MedCalc v. $12.2^{\circ}$ for Windows (MedCalc software co, Belgium, EU) and Statistica $10^{\circ}$ for Windows (StatSoft inc, Tulsa, OK, USA).

\section{Results}

\section{Studied sample}

The study included 255 cases referred for CMR a 3.75year period, covering 1975 exams. Control group of patients $(n=155 ; 60.8 \%)$ included cases with no structural heart disease and those with non-ischemic cardiomyopathy other than LVNC. Patients with LVNC that fulfilled all three CMR criteria (thinning, ratio and share, as previously explained) were included, making sample of one hundred (39.2\%) consecutive patients $(5.1 \%$ prevalence of total exams in the studied period) From those patients, only 2 cases were confirmed by addition of clinical criteria (not having $>20 \%$ of trabeculations in the left ventricular mass despite having $\mathrm{NC} / \mathrm{C}>2.3: 1)$. Fifty-six cases $(36.1 \%)$ from the control group had clinically suspicious, but not clearly defined LVNC. Detailed data on patients and studied groups are presented in Table 1.

\section{Late gadolinium enhancement}

LGE was found in 133 patients (52.2\%) as follows: focal mid-ventricular (MV) type was found in 35 (26.3\%) cases, linear MV in $83(62.4 \%)$ and diffuse LGE in 15 (11.3\%) cases. The most common type of LGE in LVNC was linear MV, located in basal $(n=44)$ and basal/middle sections $(n=8)$ of the left ventricle, whilst the patients with no structural heart disease most commonly had no LGE imbibition.

Correlations between LGE, geometry and LVNC diagnostic parameters for the studied sample of cases are presented in Table 2.

Existence of LGE was found in cases with LVEF $\leq 49.2 \%$ using receiver operating characteristic curve analysis, with sensitivity of $50.8 \%$ (95\%-CI:41.6-59.2, specificity of $85.1 \%$ (95\%-CI:77.5-90.9), +likelihood ratio (+LR) of 3.4 (95\%-CI:2.8-4.1), -LR of 0.6 (0.4-0.9), area under curve (AUC) of 0.674 (95\%-CI: 0.612-0.731, $p<0.001$.

Using binomial regression analyzes, existence of LGE was significantly connected with following studied parameters: LV-myocardial mass $=1.01$ (95\%-CI: $1.00-1.02), p=$ $0.048 ; \quad$ LVEF $=0.97 \quad(95 \%-C I: 0.95-0.98), \quad p=0.002$ and 
Table 1 Differences between LVNC patients and controls

\begin{tabular}{|c|c|c|c|}
\hline & Controls & LVNC & Chi square \\
\hline & $n=155$ & $n=100$ & \\
\hline Male & 95 (61.3\%) & $56(56.0 \%)$ & 0.401 \\
\hline Female & 60 (38.7\%) & 44 (44.0\%) & \\
\hline LVEF $<50 \%$ & 37 (23.9\%) & 48 (48.0\%) & $<0.001$ \\
\hline OK & 79 (51.0\%) & $0(0.0 \%)$ & $<0.001$ \\
\hline LVNC & $0(0.0 \%)$ & $100(100.0 \%)$ & \\
\hline DCM & $27(17.4 \%)$ & $0(0.0 \%)$ & \\
\hline $\mathrm{HCM}$ & 33 (21.3\%) & $0(0.0 \%)$ & \\
\hline ARVD & $5(3.2 \%)$ & $0(0.0 \%)$ & \\
\hline $\mathrm{HHD}$ & $6(3.9 \%)$ & $0(0.0 \%)$ & \\
\hline \multirow[t]{3}{*}{ MCD } & $5(3.2 \%)$ & $0(0.0 \%)$ & \\
\hline & Controls & LVNC & Mann-Whitney \\
\hline & $n=155$ & $n=100$ & \\
\hline Age (years) & $48.2 \pm 15.4$ & $45.5 \pm 17.8$ & 0.180 \\
\hline $\mathrm{LA}(\mathrm{cm} 2)$ & $26.9 \pm 8.6$ & $25.6 \pm 6.4$ & 0.333 \\
\hline $\mathrm{RA}(\mathrm{cm} 2)$ & $24.6 \pm 7.6$ & $23.1 \pm 6.2$ & 0.155 \\
\hline LVEDD (cm) & $5.4 \pm 1.0$ & $5.7 \pm 0.8$ & $<0.001$ \\
\hline IVS (cm) & $1.2 \pm 0.4$ & $1.0 \pm 0.2$ & 0.001 \\
\hline $\mathrm{EDV}(\mathrm{mL})$ & $160.3 \pm 78.7$ & $169.4 \pm 61.3$ & 0.036 \\
\hline ESV (mL) & $79.4 \pm 76.4$ & $95.9 \pm 69.1$ & $<0.001$ \\
\hline $\mathrm{SV}(\mathrm{mL})$ & $84.4 \pm 45.7$ & $78.5 \pm 25.6$ & 0.244 \\
\hline LVEF (\%) & $55.3 \pm 15.2$ & $48.2 \pm 12.7$ & $<0.001$ \\
\hline MM (gram) & $122.2 \pm 48.5$ & $106.0 \pm 36.3$ & 0.006 \\
\hline
\end{tabular}

LVNC Left ventricular non compaction, OK No structural heart disease, DCM Dilative cardiomyopathy, HCM Hypertophic cardiomyopathy, ARVD Arrhythmogenic right ventricle disease, $H H D$ Hypertensive heart disease, MCD Myocarditis, $L V E D D$ $(\mathrm{cm})$ Left ventricle end diastolic dimension in 4-chamber view, IVS (cm) Interventricular septum thickness in 4-chamber view, LA $(\mathrm{cm} 2)$ Left atrial area in square centimeters in 4-chamber view, LVEF (\%) Left ventricle ejection fraction, EDV ( $m L$ ) End diastolic volume, ESV ( $m L$ ) End systolic volume, $S V(m L)$ Stroke volume, LVEF (\%) Left ventricle ejection fraction, MM (gram) Myocardial mass in end-diastole. Data shown as numbers with percentages or means with standard deviations (SD). Significant values are outlined in bold

Max-Min-EDDR 1.01 (95\%-CI: 1.00-28.99), $p=0.045$, while the $\mathrm{NC} / \mathrm{C}$ was not significant.

Differences between existence of LGE in patients with LVNC and controls, with further sub-analyzes of type of LGE are presented in Tables 3 and 4.

\section{Geometry of the left ventricle}

Max-Min-EDDR was $1.11 \pm 0.09$ (95\%-CI: 1.01-1.26), with significant difference between the patients with LVNC and controls, $1.17 \pm 0.09$ vs. $1.06 \pm 0.05, p<0.001$. Similarly, Max-Min-EDDR of LVNC was significantly different among all the sub-studied control groups (no structural heart disease, $1.06 \pm 0.04$; hypertensive heart disease, $1.07 \pm 0.03$; dilative cardiomyopathy $1.05 \pm 0.04$; hypertrophic cardiomyopathy $1.08 \pm 0.08$; ARVD $1.07 \pm$ 0.03 and myocarditis $1.05 \pm 0.01), p<0.001$. In addition, Max-Min-EDDR was significantly different between the patients with no LGE imbibition and controls, $1.09 \pm$ 0.08 vs. $1.12 \pm 0.09$, respectively, $p=0.039$. Figure 1 . shows examples of geometric eccentricity in a patient with LVNC and two other cardiomyopathies. Correlations of Max-Min-EDDR and studied diagnostic parameters is presented in Table 2.

Model of receiver operating characteristic curve analysis of Max-Min-EDDR for detection of systolic function impairment (defined with $\operatorname{LVEF}<50 \%$ ) was not significant $(\mathrm{AUC}=0.559 ; p=0.121)$.

\section{Diagnostic utility of CMR parameters for LVNC}

Receiver operating characteristic (ROC) curve analysis was used for diagnostic utility of the studied CMR parameters for establishing a LVNC diagnosis, in which $\mathrm{NC} / \mathrm{C}>2.3: 1$ and $\mathrm{NC}>20 \% \mathrm{LV}$ were considered as the gold standard.

Existing LGE had sensitivity of 52.5\% (95\%-CI:42.3-62.5), specificity of $80.4 \%$ (95\%-CI:73.2-86.5), +LR of 2.7 (95\%CI:2.2-3.3), -LR of $0.6(0.4-0.9)$, area under curve (AUC) of 0.664 (95\%-CI: $0.603-0.722) ; p<0.001$.

MaxMinEDDR being $>1.10$ (i.e. exactly 1.0975) had sensitivity of $95.0 \%$ (95\%-CI:88.7-98.4), specificity of 82.6\% (95\%-CI:75.7-88.2), +LR of 5.0 (95\%-CI:5.0-5.9), -LR of $0.1(0.02-0.2)$, area under curve (AUC) of 0.917 (95\%-CI: 0.876-0.948); $p<0.001$.

Comparison of ROC curves for the studied diagnostic parameters of LVNC was as follows: ROC-AUC (NC > $20 \% \mathrm{LV})=0.985$ (95\%-CI: 0.961-0.996), ROC-AUC (NC/ C $>2.3: 1)=0.983 \quad(95 \%-C I: \quad 0.959-0.995), \quad$ ROC-AUC $($ MaxMinEDDR $)=0.909 \quad(95 \%-C I: \quad 0.866-0.941) \quad$ and ROC-AUC (linear-MV-LGE) $=0.664 \quad(95 \%$-CI:0.6030.722). There was also a significant difference between ROC AUC for LGE and eccentricity, $\triangle \mathrm{AUC0.244}$ (95\%CI:0.175-0.314), $p<0.001$, as shown in Fig. 2.

\section{Discussion}

This study analyzed the existence and morphology of LGE imbibitions, their connections with left ventricle geometry, as well as their diagnostic utility for LVNC. The "gold standard" for LVNC diagnosis using CMR was defined by thinning of compact LV wall, $\mathrm{NC} / \mathrm{C}>$ 2.3:1 and $\mathrm{NC}>20 \% \mathrm{LV}$ [11]. In these terms, our study used substantially different methodology from several recently published registry based studies that found higher prevalence of trabeculations, but without serious prognostic implications, most likely due to not having a primary objective of LVNC diagnostics per se, different inclusion criteria and not assessing the existence of LGE [20]. In addition, impairment of systolic function of the left ventricle in our study was also more congruent with previous reports [21].

Nearly a half of the studied patients with LVNC had LGE imbibition, where the latter attained specificity and 
Table 2 Correlations of LGE, geometry and other diagnostic parameters in the studied population sample $(n=255)$

\begin{tabular}{|c|c|c|c|c|c|c|c|c|c|c|c|c|}
\hline & & LVNC & $\mathrm{NC} / \mathrm{C}>2.3: 1$ & $\mathrm{NC}>20 \% \mathrm{LV}$ & LGE & LVEF $<50 \%$ & $C$ & $\mathrm{NC}$ & $\mathrm{NC} / \mathrm{C}$ & MIN EDD & MAX EDD & MaxMin EDDR \\
\hline \multirow[t]{2}{*}{ LVNC } & Rho CC & NA & 0.888 & 0.960 & 0.140 & 0.234 & -0.668 & 0.560 & 0.795 & -0.079 & 0.234 & 0.689 \\
\hline & $p$ & & $<0.001$ & $<0.001$ & 0.025 & $<0.001$ & $<0.001$ & $<0.001$ & $<0.001$ & 0.206 & $<0.001$ & $<0.001$ \\
\hline \multirow[t]{2}{*}{ MNC } & Rho CC & 0.032 & 0.240 & NA & 0.066 & 0.114 & -0.258 & 0.496 & 0.575 & 0.032 & 0.061 & 0.093 \\
\hline & $p$ & 0.690 & 0.003 & & 0.414 & 0.156 & 0.001 & $<0.001$ & $<0.001$ & 0.693 & 0.454 & 0.249 \\
\hline \multirow[t]{2}{*}{ Age (years) } & Rho CC & -0.094 & -0.038 & -0.095 & 0.137 & 0.110 & 0.118 & 0.061 & -0.053 & 0.115 & 0.100 & -0.087 \\
\hline & $p$ & 0.136 & 0.544 & 0.132 & 0.029 & 0.080 & 0.060 & 0.333 & 0.395 & 0.066 & 0.110 & 0.168 \\
\hline \multirow[t]{2}{*}{ LA (cm2) } & Rho CC & -0.063 & -0.087 & -0.060 & 0.143 & 0.275 & 0.210 & 0.075 & -0.085 & 0.522 & 0.506 & -0.070 \\
\hline & $p$ & 0.314 & 0.165 & 0.338 & 0.023 & $<0.001$ & 0.001 & 0.233 & 0.176 & $<0.001$ & $<0.001$ & 0.268 \\
\hline \multirow[t]{2}{*}{$\mathrm{EDV}(\mathrm{mL})$} & Rho CC & 0.128 & 0.074 & 0.130 & 0.173 & 0.426 & 0.058 & 0.188 & 0.082 & 0.729 & 0.750 & 0.008 \\
\hline & $p$ & 0.042 & 0.238 & 0.039 & 0.006 & $<0.001$ & 0.359 & 0.003 & 0.192 & $<0.001$ & $<0.001$ & 0.905 \\
\hline \multirow[t]{2}{*}{ ESV (mL) } & Rho CC & 0.255 & 0.210 & 0.265 & 0.224 & 0.666 & -0.041 & 0.294 & 0.218 & 0.685 & 0.726 & 0.059 \\
\hline & $p$ & $<0.001$ & 0.001 & $<0.001$ & $<0.001$ & $<0.001$ & 0.512 & $<0.001$ & $<0.001$ & $<0.001$ & $<0.001$ & 0.344 \\
\hline \multirow[t]{2}{*}{ LVEDD $(\mathrm{cm})$} & Rho CC & 0.209 & 0.182 & 0.226 & 0.206 & 0.520 & 0.006 & 0.318 & 0.196 & 0.656 & 0.689 & 0.043 \\
\hline & $p$ & 0.001 & 0.004 & $<0.001$ & 0.001 & $<0.001$ & 0.930 & $<0.001$ & 0.002 & $<0.001$ & $<0.001$ & 0.491 \\
\hline \multirow[t]{2}{*}{ IVS (cm) } & Rho CC & -0.196 & -0.169 & -0.220 & 0.262 & 0.057 & 0.377 & -0.026 & -0.251 & 0.202 & 0.130 & -0.137 \\
\hline & $p$ & 0.002 & 0.007 & $<0.001$ & $<0.001$ & 0.369 & $<0.001$ & 0.676 & $<0.001$ & 0.001 & 0.037 & 0.029 \\
\hline \multirow[t]{2}{*}{ LVEF } & Rho CC & -0.313 & -0.309 & -0.332 & -0.301 & -0.809 & 0.154 & -0.330 & -0.311 & -0.507 & -0.570 & -0.117 \\
\hline & $p$ & $<0.001$ & $<0.001$ & $<0.001$ & $<0.001$ & $<0.001$ & 0.014 & $<0.001$ & $<0.001$ & $<0.001$ & $<0.001$ & 0.062 \\
\hline \multirow[t]{2}{*}{ MM (gram) } & Rho CC & -0.157 & -0.190 & -0.168 & 0.202 & 0.253 & 0.395 & 0.025 & -0.222 & 0.554 & 0.498 & -0.144 \\
\hline & $p$ & 0.012 & 0.002 & 0.007 & 0.001 & $<0.001$ & $<0.001$ & 0.687 & $<0.001$ & $<0.001$ & $<0.001$ & 0.021 \\
\hline
\end{tabular}

LVNC Left ventricular non compaction, NC/C > 2.3:1 NC to C ratio > 2.3:1 - non-compact to compact layers thickness ratio, $L G E$ Late gadolinium enhancement, NC $>20 \%$ LV Non-compact myocardium proportion $>20 \%$ of the left ventricle, EDD End diastolic dimension, Max maximal, Min minimal, MaxMinEDDR ratio of maximal to minimal EDD, MNC Marginal non-compaction (clinical suspicions of LVNC), NA Not applicable, LVEDD (cm) Left ventricle end diastolic dimension in 4-chamber view, IVS (cm) Interventricular septum thickness in 4-chamber view, LA (cm2) Left atrial area in square centimeters in 4-chamber view, LVEF (\%) Left ventricle ejection fraction, EDV $(\mathrm{mL})$ End diastolic volume, ESV $(m L)$ End systolic volume, $L V E F(\%)$ Left ventricle ejection fraction, $M M(g r a m)$ Myocardial mass in end-diastole. Significant correlations presented in bold

sensitivity of $55-60 \%$ as a diagnostic parameter. Proportion of patients with LGE in our study was in line with previous reports on the left ventricle non-compaction cardiomyopathy [22]. The most common pattern of LGE in LVNC was linear midventricular, followed by none, while only a small number of cases had diffuse imbibitions [22]. Interestingly, linear midventricular LGE in patients with non-compaction was located in basal and basal to middle sections of the left ventricle, whilst mid apical regions with the most pronounced trabeculations did not show clinically significant proportion of late imbibitions. Linear midventricular type of LGE was the most common LGE type in patients with dilative cardiomyopathy, focal type LGE was the most prevalent in patients with hypertrophic cardiomyopathy, while the patients with no structural heart disease predominantly had no late imbibitions [23]. There were no significant differences in LGE imbibition between the cases with suspected LVNC and the remaining part of the control group. The latter reveled the shortcomings of late gadolinium imbibition as a diagnostic tool for differentiating between the cases with increased trabeculations of the left ventricle suspected of LVNC. However, it is worthwhile to note that the difference of LGE imbibition was significant to cases with overt LVNC [24]. Existence of LGE correlated only to a mild degree with the age of patients, and there was a strong inverse correlation with systolic function of the left ventricle [25]. The former seems to represent a time-dependent degenerative process, and support the fact of overall underprivileged prognostic course in patients with heart failure [26]. Critical cut-off point to the left ventricle ejection fraction for existence of LGE was found to be set at $\leq 49.2 \%$, as an intermediate-grade sensitive and high-grade specific sign of systolic impairment [24]. Existence of LGE imbibition in the left ventricle was connected with increased end diastolic dimension, interventricular septum thickness and enlarged atrial volumes, which could imply further diagnostic-prognostic value of this parameter [27]. Regarding the LV-volumetrics, LGE was in mild correlation with end diastolic volume, end systolic volume and LV-myocardial mass. Changes in cardiomyocytes, which made them susceptible for late gadolinium imbibition, consequentially increased size of the left ventricle and atria, while systolic function decreased, representing the point of down turning of the Frank-Starling curve and value of LGE as the initial diagnostic landmark of the failing heart $[28,29]$. 
Table 3 Principal characteristics and differences in the studied sample, depending on the existence and type of late gadolinium enhancement

\begin{tabular}{|c|c|c|c|c|c|c|c|c|}
\hline & No LGE & LGE & & No LGE & Focal MV & Linear MV & Diffuse & Kruskal \\
\hline & n (\%) & n (\%) & square & n (\%) & n (\%) & n (\%) & n (\%) & \\
\hline Male & 64 (52.5\%) & 87 (65.4\%) & 0.031 & $64(52.5 \%)$ & 27 (77.1\%) & 49 (59.0\%) & $11(73.3 \%)$ & 0.038 \\
\hline Female & $58(47.5 \%)$ & 46 (34.6\%) & & $58(47.5 \%)$ & 8 (22.9\%) & 34 (41.0\%) & 4 (26.7\%) & \\
\hline OK & 67 (54.9\%) & $12(9.0 \%)$ & $<0.001$ & 67 (54.9\%) & $3(8.6 \%)$ & 7 (8.4\%) & 2 (13.3\%) & $<0.001$ \\
\hline LVNC & 40 (32.8\%) & 60 (45.1\%) & & 40 (32.8\%) & $4(11.4 \%)$ & $52(62.7 \%)$ & 4 (26.7\%) & \\
\hline DCM & $4(3.3 \%)$ & $23(17.3 \%)$ & & 4 (3.3\%) & $3(8.6 \%)$ & 18 (21.7\%) & 2 (13.3\%) & \\
\hline $\mathrm{HCM}$ & $2(1.6 \%)$ & 31 (23.3\%) & & $2(1.6 \%)$ & 22 (62.9\%) & $3(3.6 \%)$ & $6(40.0 \%)$ & \\
\hline ARVD & $4(3.3 \%)$ & $1(0.8 \%)$ & & 4 (3.3\%) & $0(0.0 \%)$ & $1(1.2 \%)$ & $0(0.0 \%)$ & \\
\hline $\mathrm{HHD}$ & $4(3.3 \%)$ & $2(1.5 \%)$ & & 4 (3.3\%) & $0(0.0 \%)$ & $2(2.4 \%)$ & $0(0.0 \%)$ & \\
\hline MCD & $1(0.8 \%)$ & 4 (3.0\%) & & $1(0.8 \%)$ & $3(8.6 \%)$ & $0(0.0 \%)$ & $1(6.7 \%)$ & \\
\hline LVEF < 50\% & 20 (16.4\%) & 65 (48.9\%) & $<0.001$ & $20(16.4 \%)$ & 13 (37.1\%) & 48 (57.8\%) & $4(26.7 \%)$ & $<0.001$ \\
\hline$N C: C>2.3: 1$ & 40 (32.8\%) & 67 (50.4\%) & 0.005 & 40 (32.8\%) & $6(17.1 \%)$ & 56 (67.5\%) & 5 (33.3\%) & $<0.001$ \\
\hline LVNC & 40 (32.8\%) & 60 (45.1\%) & $<0.001$ & 40 (32.8\%) & $4(11.4 \%)$ & 52 (63.9\%) & 4 (26.7\%) & $<0.001$ \\
\hline MNC & 27 (32.9\%) & 29 (39.7\%) & 0.410 & 27 (32.9\%) & 10 (32.3\%) & 14 (45.2\%) & 5 (45.5\%) & 0.560 \\
\hline MCE & 26 (21.3\%) & 59 (44.4\%) & $<0.001$ & $26(21.3 \%)$ & 17 (48.6\%) & 33 (39.8\%) & $9(60.0 \%)$ & 0.001 \\
\hline$N C>20 \%$ LV & 39 (32.0\%) & 59 (44.4\%) & 0.047 & 39 (32.0\%) & 3 (8.6\%) & 52 (62.7\%) & $4(26.7 \%)$ & $<0.001$ \\
\hline
\end{tabular}

LGE late gadolinium enhancement, MV Midventricular, LVNC Left ventricular non compaction, OK No structural heart disease, DCM Dilative cardiomyopathy, HCM Hypertophic cardiomyopathy, ARVD Arrhythmogenic right ventricle disease, HHD Hypertensive heart disease, MCD Myocarditis, LVEF (\%) Left ventricle ejection fraction, NC to C ratio Non-compact to compact layers thickness ratio, MNC Marginal non-compaction (clinical suspicion of LVNC), MCE Multiple cardiomyopathy elements, NC > 20\%LV Non-compact myocardium proportion $>20 \%$ of the left ventricle. Significant values outlined in bold

Cases with LVNC displayed structural rearrangements in terms of geometric eccentricity of mid to apical short axis sections, affected with non-compaction [30]. Eccentricity of the left ventricle was in significant and powerful correlation with LVNC diagnosis and its diagnostic parameters. MaxMinEDDR did not correlate in cases with clinical suspicion of LVNC or those with multiple cardiomyopathy elements, underscoring further diagnostic value of geometric changes for LVNC. In addition, correlation of Max-Min-EDDR with LVNC diagnostic criteria attained powerful synergy, while its constituents, taken individually had only mild or insignificant correlations. Difference between maximal and minimal end diastolic ratio of over $10 \%$ had $95 \%$ sensitivity and $83 \%$ specificity for LVNC, making it a very promising innovative diagnostic parameter. Patients with LGE imbibitions also had significantly different eccentricity of the left ventricle, however in close numeric range to controls (without LGE imbibition). Correlations of LGE with the geometry of the left ventricle were significant, though mild, leading to an assumption that LGE is not in a straightforward relation with geometry, and that it is more likely secondarily changed due to infrastructural rearrangements of the left ventricle which consequently ended up as geometric eccentricity [31, 32]. In head to head settings, MaxMinEDDR was shown to be a superior diagnostic parameter than LGE, of similar diagnostic efficiency as the $\mathrm{NC} / \mathrm{C}$ ratio or $\mathrm{NC}$ affecting more than $20 \%$ of the left ventricle, with the last two considered as among the best currently available evidence for diagnosing the LVNC $[3,12]$.

Geometry of the left ventricle was found to be connected with functional prognostic parameters such as systolic function or left atrial size [27]. The latter implies that local infrastructural alternations in terms of noncompaction eventually cause changes in the structure and function beyond the left ventricle, which could serve as a diagnostic landmark of the turning point of the failing heart. Systolic function of the left ventricle correlated with maximal and minimal end diastolic diameters in mid apical short axis slices, but not with MaxMinEDDR. This was further confirmed as a clinically insignificant diagnostic parameter of systolic dysfunction in ROC analysis. Based on those results, assumptions could be made that whilst systolic function of the left ventricle in patients with LVNC tends to be decreased, the initial disorder is probably hidden among the structural changes of non-compact myocardium, and eminently ends up with different grade of systolic dysfunction. There seems to be sequential relation, where systolic dysfunction lags in phase to infrastructural changes caused by non-compact myocardium and changes of geometry.

One must point out that these are preliminary investigations, focused on diagnostic utility of a single imaging modality scrutinized according to guidelines-based diagnostic criteria that are not perfect. The study was 
Table 4 Characteristics of myocardial layers and left ventricle geometry in dependence of the existence of late gadolinium enhancement

\begin{tabular}{|c|c|c|c|c|c|c|c|c|}
\hline & \multirow{2}{*}{$\begin{array}{l}\text { No LGE } \\
\text { Mean } \pm \text { SD }\end{array}$} & \multirow{2}{*}{$\begin{array}{l}\text { LGE } \\
\text { Mean } \pm S D\end{array}$} & \multirow{2}{*}{$\begin{array}{l}\text { Mann- } \\
\text { Whitney }\end{array}$} & \multirow{2}{*}{$\begin{array}{l}\text { No LGE } \\
\text { Mean } \pm S D\end{array}$} & \multirow{2}{*}{$\begin{array}{l}\text { Focal MV } \\
\text { Mean } \pm \text { SD }\end{array}$} & \multirow{2}{*}{$\begin{array}{l}\text { Linear MV } \\
\text { Mean } \pm \text { SD }\end{array}$} & \multirow{2}{*}{$\begin{array}{l}\text { Diffuse } \\
\text { Mean } \pm \text { SD }\end{array}$} & \multirow{2}{*}{$\begin{array}{l}\text { Kruskal } \\
\text { Wallis }\end{array}$} \\
\hline & & & & & & & & \\
\hline Age (years) & $44.9 \pm 15.3$ & $49.1 \pm 17.2$ & 0.029 & $44.9 \pm 15.3$ & $50.8 \pm 16.8$ & $49.0 \pm 17.7$ & $46.1 \pm 16.3$ & $<0.001$ \\
\hline LVEDD $(\mathrm{cm})$ & $5.31 \pm 0.66$ & $5.73 \pm 1.06$ & 0.001 & $5.31 \pm 0.66$ & $5.39 \pm 0.83$ & $5.95 \pm 1.09$ & $5.33 \pm 1.09$ & 0.124 \\
\hline IVS (cm) & $0.98 \pm 0.17$ & $1.19 \pm 0.43$ & $<0.001$ & $0.98 \pm 0.17$ & $1.50 \pm 0.55$ & $1.04 \pm 0.22$ & $1.35 \pm 0.57$ & $<0.001$ \\
\hline $\mathrm{RV}(\mathrm{cm})$ & $3.71 \pm 0.68$ & $3.58 \pm 0.79$ & 0.230 & $3.71 \pm 0.68$ & $3.68 \pm 0.69$ & $3.57 \pm 0.84$ & $3.43 \pm 0.70$ & 0.424 \\
\hline LA (cm2) & $24.88 \pm 5.89$ & $27.89 \pm 8.95$ & 0.023 & $24.88 \pm 5.89$ & $28.11 \pm 7.21$ & $28.06 \pm 9.78$ & $26.40 \pm 8.10$ & 0.105 \\
\hline $\mathrm{RA}(\mathrm{cm} 2)$ & $22.82 \pm 6.21$ & $25.14 \pm 7.68$ & 0.016 & $22.82 \pm 6.21$ & $25.09 \pm 6.57$ & $25.23 \pm 7.83$ & $24.80 \pm 9.57$ & 0.064 \\
\hline $\mathrm{EDV}(\mathrm{mL})$ & $149.4 \pm 45.4$ & $177.5 \pm 88.5$ & 0.006 & $149.4 \pm 45.4$ & $156.9 \pm 50.3$ & $190.4 \pm 103.2$ & $153.1 \pm 47.8$ & 0.014 \\
\hline ESV (mL) & $66.4 \pm 32.5$ & $103.9 \pm 94.2$ & $<0.001$ & $66.4 \pm 32.5$ & $79.4 \pm 52.3$ & $120.8 \pm 110.0$ & $67.3 \pm 39.1$ & $<0.001$ \\
\hline $\mathrm{SV}(\mathrm{mL})$ & $88.1 \pm 49.6$ & $76.6 \pm 25.4$ & 0.008 & $88.1 \pm 49.6$ & $77.0 \pm 21.6$ & $74.7 \pm 26.6$ & $85.8 \pm 26.0$ & 0.020 \\
\hline LVEF (\%) & $57.1 \pm 8.8$ & $48.2 \pm 17.4$ & $<0.001$ & $57.1 \pm 8.8$ & $53.3 \pm 17.5$ & $44.4 \pm 16.6$ & $57.6 \pm 16.0$ & $<0.001$ \\
\hline MM (gram) & $105.8 \pm 32.7$ & $125.2 \pm 51.9$ & 0.001 & $105.8 \pm 32.7$ & $139.2 \pm 38.0$ & $119.5 \pm 57.3$ & $125.2 \pm 44.1$ & $<0.001$ \\
\hline C- thickness $(\mathrm{cm})$ & $0.65 \pm 0.20$ & $0.67 \pm 0.24$ & 0.853 & $0.65 \pm 0.20$ & $0.84 \pm 0.24$ & $0.59 \pm 0.21$ & $0.73 \pm 0.23$ & $<0.001$ \\
\hline NC- thickness $(\mathrm{cm})$ & $1.10 \pm 0.46$ & $1.18 \pm 0.48$ & 0.025 & $1.10 \pm 0.46$ & $0.96 \pm 0.51$ & $1.31 \pm 0.42$ & $1.01 \pm 0.53$ & $<0.001$ \\
\hline $\mathrm{NC}$ to $\mathrm{C}$ ratio & $1.99 \pm 1.29$ & $2.14 \pm 1.20$ & 0.127 & $1.99 \pm 1.29$ & $1.30 \pm 0.85$ & $2.58 \pm 1.09$ & $1.65 \pm 1.27$ & $<0.001$ \\
\hline Minimal MA EDD (cm) & $5.16 \pm 0.66$ & $5.43 \pm 1.02$ & 0.040 & $5.16 \pm 0.66$ & $5.19 \pm 0.83$ & $5.56 \pm 1.14$ & $5.25 \pm 0.39$ & 0.130 \\
\hline Maximal MA EDD $(\mathrm{cm})$ & $5.63 \pm 0.75$ & $6.04 \pm 1.06$ & $<0.001$ & $5.63 \pm 0.75$ & $5.68 \pm 0.69$ & $6.24 \pm 1.21$ & $5.77 \pm 0.48$ & $<0.001$ \\
\hline Max to Min EDD Ratio & $1.09 \pm 0.08$ & $1.12 \pm 0.09$ & 0.039 & $1.09 \pm 0.08$ & $1.10 \pm 0.11$ & $1.13 \pm 0.08$ & $1.10 \pm 0.11$ & 0.005 \\
\hline LV trabeculations \% & $14.7 \pm 11.2$ & $17.4 \pm 12.2$ & 0.117 & $14.7 \pm 11.2$ & $8.2 \pm 7.7$ & $21.7 \pm 11.7$ & $13.4 \pm 10.8$ & $<0.001$ \\
\hline
\end{tabular}

LGE Late gadolinium enhancement, MV Midventricular, LVNC Left ventricular non compaction; LV-NC \% - left ventricle non-compaction proportion in total mass of the left ventricle, C-Compact $(\mathrm{cm})$ Thickness of solid myocardium, NC-non-compact $(\mathrm{cm})$ Thickness of trabeculated part i.e. non-compaction myocardium, LVEDD $(\mathrm{cm})$ Left ventricle end diastolic dimension in 4-chamber view, IVS $(\mathrm{cm})$ Interventricular septum thickness in 4-chamber view, $R V$ ( $\mathrm{cm}$ ) Right ventricle end diastolic dimension in 4-chamber view, LA \& RA (cm2) Left and right atrial area in square centimeters in 4-chamber view, LVEF (\%) Left ventricle ejection fraction, EDV ( $m L$ ) End diastolic volume, ESV ( $\mathrm{mL}$ ) End systolic volume, SV ( $\mathrm{mL}$ ) Stroke volume, MM (gram) Myocardial mass in end-diastole, MA Mid to apical slices in 6-mm pile stack of short axes, EDD End diastolic dimension, Max Maximal, Min Minimal. Data shown as mean with standard deviations (SD). Statistically significant values $(p<0.05)$ presented in bold

performed in the only large-volume tertiary center specialized for CMR in our country, on a selected population of cases with cardiovascular diseases and previous cardiovascular diagnostic and/or therapeutic workup. Retrospective study settings and selection of controls also might conceal limitations. Prospective validation, using multicentric settings, with inclusion of further clinical parameters, such as patients' comorbidities and follow up of major adverse event rates is necessary to increase reproducibility.

\section{Conclusion}

In conclusion, late gadolinium enhancement was found to be frequently expressed in patients with LVCN. These

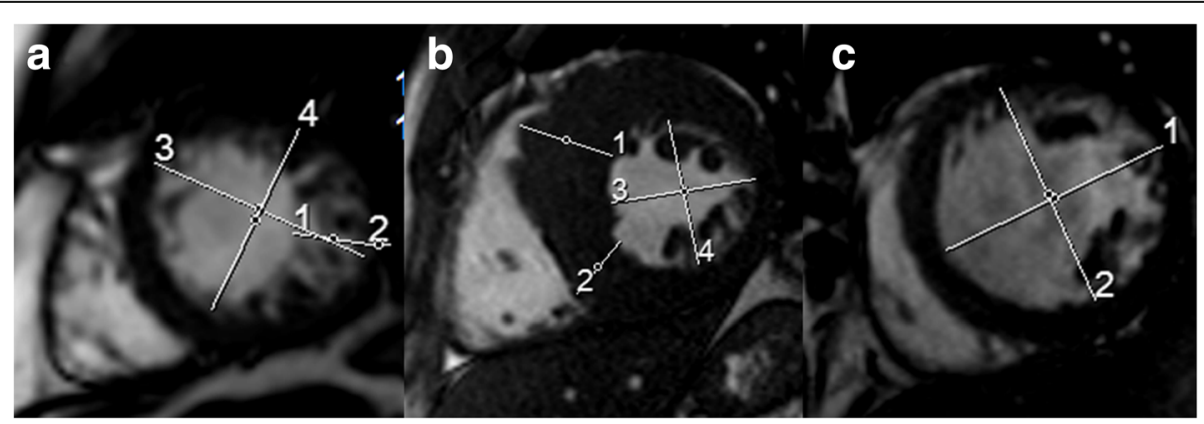

Fig. 1 Geometry of the left ventricle in various heart diseases Data labels: a Case of patient with left ventricular non-compaction and trabeculations existing on $35.3 \%$ of the left ventricle, ejection fraction of $52 \%$ and confirmed non-compaction; short axis cine at end diastole (ED), marked white line showing: 1) NC-layer thickness $2.22 \mathrm{~cm}$, 2) C-layer thickness $0.57 \mathrm{~cm}$, with NC/C =3.9. 3) Maximal ED dimension $6.40 \mathrm{~cm}$, 4) Minimal EDD $5.59 \mathrm{~cm}$, giving MaxMinEDDR = 1.14; (b) patient with hypertrophic cardiomyopathy: 1) $3.41 \mathrm{~cm}, 2) 2.40 \mathrm{~cm}$, 3) $5.17 \mathrm{~cm}$, 4) $5.16 \mathrm{~cm}$ i.e. Max-Min EDDR=1.00; (c) patient with dilative cardiomyopathy: 1) $7.20 \mathrm{~cm}$, 2) $6.99 \mathrm{~cm}$ i.e. Max-Min EDDR $=1.03$ 


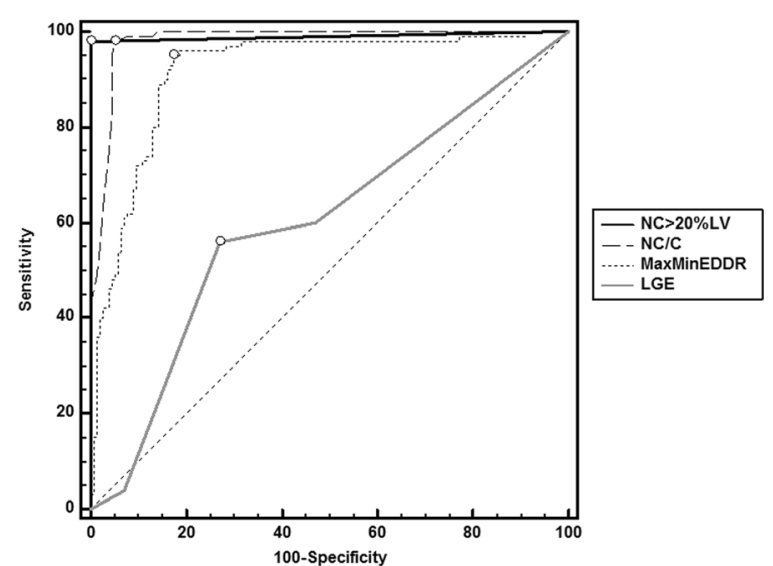

Fig. 2 Comparison of receiver operating characteristic (ROC) curve analysis for LVNC diagnostic parameters. Data labels: LGE - late gadolinium enhancement; NC/C - non-compact to compact layer thickness ratio; NC\% - non-compact myocardium percentage in total left ventricle mass; MaxMinEDDR - maximal to minimal end diastolic diameter (EDD) ratio; C - compact layer thickness; NC - non-compact layer thickness; - showing value with the highest Youden index

imbibitions were of insufficiently defined power to be used as a specific diagnostic parameter, in the context of LVNC. On the other hand, LGE was found to be a relatively valuable diagnostic landmark of complex infrastructural and functional changes within the failing heart. It correlated only mildly with left ventricle geometry in dedicated regions, where the latter was found to be a very promising novel LVNC diagnostic parameter. Geometric eccentricity associated with LVNC had a fine grade of diagnostic utility to tell apart the cases with non-ischemic cardiomyopathies from those with structurally normal heart. Segmental eccentricity was also in correlation with infrastructural and functional changes that even stretched beyond mid-apical regions, i.e. to the whole left ventricle and both atria. Changes of left ventricle geometry in addition to tissue characterization by late gadolinium imbibition seem to have potential for further contribution to the diagnostic imaging of the failing heart.

\section{Abbreviations}

\%: Percentages; +LR: Positive likelihood ratio; 95\%Cl: 95\%-confidence intervals; ARVD: Arrhythmogenic right ventricle dysplasia; AUC: Area under the curve; CMR: Cardiac magnetic resonance imaging; Compact (cm): End diastolic thickness of solid myocardial layer; DB: dark blood preparation; DCM: Dilative cardiomyopathy; ED: End diastolic; EDD: End diastolic dimension; EDV (mL): End diastolic volume in milliliters; ESV (mL): End systolic volume; Gd: Gadolinium; HASTE: Half-Fourier Acquisition Single-shot Turbo spin Echo; HCM: Hypertrophic cardiomyopathy; ICT2HI: Intracavital T-2-weighted hyperintensity; IVS (cm): Interventricular septum thickness in 4-chamber view; LA \& RA (cm2): Left and right atrial area in square centimeters in 4-chamber view; LGE: Late gadolinium enhancement; LR: Negative likelihood ratio; LVEDD $(\mathrm{cm})$ : left ventricle end diastolic dimension in 4-chamber view; LVEF (\%): Left ventricle ejection fraction; LVEF < 50\%: Impairment of left ventricle systolic function; LVNC: Left ventricular non compaction; MA: Mid to apical slices in 6-mm pile stack of short axes; Max: Maximal; MaxMinEDDR: Maximal over minimal end diastolic dimension ratio; Min: Minimal; MM (gram): Myocardial mass in end-diastole; $\mathrm{N}$ : Number; NC > 20\% LV: Percentage of trabeculations over $20 \%$ in the total mass of the left ventricle; NC\%LV: Left ventricle non-compaction proportion in the total mass of the left ventricle; NC:C: Non compact to compact myocardial layer thickness; NC:C: Non-compact to compact myocardial layer thickness ratio; Non-compact (cm): ED thickness of trabeculated part i.e. non-compaction myocardium; P: Statistical significance; Rho-CC: Spearman's rho correlation coefficient; ROC: Receiver operating characteristic; RV (cm): Right ventricle end diastolic dimension in 4-chamber view; SD: Standard deviations; SSFP: Steady state free precession; STIR: Short tau inversion recovery; SV (mL): Stroke volume; T2: relaxation - progressive dephasing of spinning dipoles following the $90^{\circ}$ pulse i.e. spin-spin relaxation; TSE: turbo spin echo

\section{Acknowledgements \\ None.}

\section{Funding}

This study was not financed or supported in any other way.

\section{Availability of data and materials}

Datasets used and/or analyzed in the study are available from the corresponding author on request.

\section{Authors' contributions}

$\mathrm{MB}$ - conception and design of the study, acquisition and interpretation of data, manuscript drafting; VLP - acquisition, processing and analyzes of imaging data; VIP - analysis of data, review of the manuscript; IDG \& MZ statistical analysis, acquisition and interpretation of data; SM - analysis of patient data and drafting the article; HAK - radiology data analysis and interpretation; AV - review of the manuscript. All authors agreed to be accountable for all aspects of the work in ensuring that questions related to the accuracy or integrity of any part of the work are appropriately investigated and resolved, and gave their final approval of the version to be submitted.

\section{Ethics approval and consent to participate}

This study was conducted in Thalassotherapia Opatija University hospital, associated with the Faculty of Medicine of the University of Rijeka and Faculty of Medicine of the University of Osijek. It was approved by the ethical committee of the Hospital. Patients were included after signing of an informed consent form. Study was conducted in accordance with the Declaration of Helsinki and good clinical practice principles.

\section{Consent for publication}

Not applicable

\section{Competing interests}

The authors declare they have no competing interests.

\section{Publisher's Note}

Springer Nature remains neutral with regard to jurisdictional claims in published maps and institutional affiliations.

\section{Author details}

'Department of Cardiology, University hospital "Thalassotherapia Opatija", Faculty of Medicine, University of Rijeka, M. Tita 188/1, 51440 Opatija, Croatia. ${ }^{2}$ Department of Internal medicine, Faculty of Medicine, "J.J. Strossmayer" University of Osijek, Osijek, Croatia. 'Department of Cardiology, University Hospital "Sestre Milosrdnice" Zagreb, Zagreb, Croatia.

Received: 26 August 2017 Accepted: 27 November 2017

Published online: 06 December 2017

\section{References}

1. Lofiego C, Biagini E, Pasquale F, Ferlito M, Rocchi G, Perugini E, BacchiReggiani L, Boriani G, Leone $\mathrm{O}$, Caliskan K, et al. Wide spectrum of presentation and variable outcomes of isolated left ventricular non-compaction. Heart. 2007; 93(1):65-71.

2. Oechslin EN, Attenhofer Jost CH, Rojas JR, Kaufmann PA, Jenni R. Long-term follow-up of 34 adults with isolated left ventricular noncompaction: a distinct cardiomyopathy with poor prognosis. J Am Coll Cardiol. 2000;36(2):493-500. 
3. Arbustini $E$, Weidemann F, Hall JL. Left ventricular noncompaction: a distinct cardiomyopathy or a trait shared by different cardiac diseases? J Am Coll Cardiol. 2014;64(17):1840-50.

4. Aryal MR, Badal M, Giri S, Pradhan R. Left ventricular non-compaction presenting with heart failure and intramural thrombus. BMJ case reports. 2013; 2013

5. Kodo K, Ong SG, Jahanbani F, Termglinchan V, Hirono K, InanlooRahatloo K, Ebert AD, Shukla P, Abilez OJ, Churko JM, et al. iPSC-derived cardiomyocytes reveal abnormal TGF-beta signalling in left ventricular non-compaction cardiomyopathy. Nat Cell Biol. 2016;18(10):1031-42.

6. Chebrolu LH, Mehta AM, Nanda NC. Noncompaction cardiomyopathy: the role of advanced multimodality imaging techniques in diagnosis and assessment. Echocardiography. 2017;34(2):279-89.

7. Quarta G, Papadakis M, Donna PD, Maurizi N, lacovoni A, Gavazzi A, Senni M, Olivotto I. Grey zones in cardiomyopathies: defining boundaries between genetic and iatrogenic disease. Nature reviews Cardiology. 2017;14(2):102-12.

8. Caselli S, Ferreira D, Kanawati E, Di Paolo F, Pisicchio C, Attenhofer Jost C, Spataro A, Jenni R, Pelliccia A. Prominent left ventricular trabeculations in competitive athletes: a proposal for risk stratification and management. Int J Cardiol. 2016;223:590-5.

9. Jenni R, Oechslin E, Schneider J, Attenhofer Jost C, Kaufmann PA. Echocardiographic and pathoanatomical characteristics of isolated left ventricular non-compaction: a step towards classification as a distinct cardiomyopathy. Heart. 2001;86(6):666-71.

10. Petersen SE, Selvanayagam JB, Wiesmann F, Robson MD, Francis JM, Anderson $\mathrm{RH}$, Watkins $\mathrm{H}$, Neubauer S. Left ventricular non-compaction: insights from cardiovascular magnetic resonance imaging. J Am Coll Cardiol. 2005;46(1):101-5.

11. Finsterer J, Stollberger C, Towbin JA. Left ventricular noncompaction cardiomyopathy: cardiac, neuromuscular, and genetic factors. Nature reviews Cardiology. 2017;14(4):224-37.

12. Jacquier A, Thuny F, Jop B, Giorgi R, Cohen F, Gaubert JY, Vidal V, Bartoli JM Habib G, Moulin G. Measurement of trabeculated left ventricular mass using cardiac magnetic resonance imaging in the diagnosis of left ventricular non-compaction. Eur Heart J. 2010;31(9):1098-104.

13. Grothoff M, Pachowsky M, Hoffmann J, Posch M, Klaassen S, Lehmkuhl L, Gutberlet M. Value of cardiovascular MR in diagnosing left ventricular non-compaction cardiomyopathy and in discriminating between other cardiomyopathies. Eur Radiol. 2012;22(12):2699-709.

14. Fazio G, Novo G, D'Angelo L, Visconti C, Sutera L, Grassedonio E, Galia M, Ferrara F, Midiri M, Novo S. Magnetic resonance in isolated noncompaction of the ventricular myocardium. Int J Cardiol. 2010;140(3):367-9.

15. Diwadkar S, Nallamshetty L, Rojas C, Athienitis A, Declue C, Cox C, Patel A, Chae $\mathrm{SH}$. Echocardiography fails to detect left ventricular noncompaction in a cohort of patients with noncompaction on cardiac magnetic resonance imaging. Clin Cardiol. 2017;40(6):364-9.

16. Levine YC, Matos J, Rosenberg MA, Manning WJ, Josephson ME, Buxton AE, Left ventricular sphericity independently predicts appropriate implantable cardioverter-defibrillator therapy. Heart rhythm : the official journal of the Heart Rhythm Society. 2016;13(2):490-7.

17. Marchal P, Lairez O, Cognet T, Chabbert V, Barrier P, Berry M, Mejean S, Roncalli J, Rousseau H, Carrie D, et al. Relationship between left ventricular sphericity and trabeculation indexes in patients with dilated cardiomyopathy: a cardiac magnetic resonance study. European heart journal cardiovascular Imaging. 2013;14(9):914-20.

18. Cheng H, Lu M, Hou C, Chen X, Li L, Wang J, Yin G, Chen X, Xiangli W, Cui C, et al. Comparison of cardiovascular magnetic resonance characteristics and clinical consequences in children and adolescents with isolated left ventricular non-compaction with and without late gadolinium enhancement. Journal of cardiovascular magnetic resonance : official journal of the Society for Cardiovascular Magnetic Resonance. 2015;17:44.

19. Nucifora G, Aquaro GD, Pingitore A, Masci PG, Lombardi M. Myocardial fibrosis in isolated left ventricular non-compaction and its relation to disease severity. Eur J Heart Fail. 2011;13(2):170-6.

20. Zemrak F, Ahlman MA, Captur G, Mohiddin SA, Kawel-Boehm N, Prince MR, Moon JC, Hundley WG, Lima JA, Bluemke DA, et al. The relationship of left ventricular trabeculation to ventricular function and structure over a 9.5-year follow-up: the MESA study. J Am Coll Cardiol. 2014;64(19):1971-80.

21. Habib G, Charron P, Eicher JC, Giorgi R, Donal E, Laperche T, Boulmier D, Pascal C, Logeart D, Jondeau G, et al. Isolated left ventricular non-compaction in adults: clinical and echocardiographic features in 105 patients. Results from a French registry. Eur J Heart Fail. 2011;13(2):177-85.
22. Wan J, Zhao S, Cheng H, Lu M, Jiang S, Yin G, Gao X, Yang Y. Varied distributions of late gadolinium enhancement found among patients meeting cardiovascular magnetic resonance criteria for isolated left ventricular non-compaction. J Cardiovasc Magn Reson. 2013;15:20.

23. Rodrigues JC, Rohan S, Ghosh Dastidar A, Harries I, Lawton CB, Ratcliffe LE, Burchell AE, Hart EC, Hamilton MC, Paton JF, et al. Hypertensive heart disease versus hypertrophic cardiomyopathy: multi-parametric cardiovascular magnetic resonance discriminators when end-diastolic wall thickness $>/=15 \mathrm{~mm}$. Eur Radiol. 2016;

24. Amzulescu MS, Rousseau MF, Ahn SA, Boileau L, de Meester de Ravenstein C, Vancraeynest D, Pasquet A, Vanoverschelde JL, Pouleur AC, Gerber BL. Prognostic impact of Hypertrabeculation and noncompaction phenotype in dilated cardiomyopathy: a CMR study. JACC Cardiovascular imaging. 2015; 8(8):934-46

25. Tandon A, Villa CR, Hor KN, Jefferies JL, Gao Z, Towbin JA, Wong BL, Mazur W, Fleck RJ, Sticka JJ, et al. Myocardial fibrosis burden predicts left ventricular ejection fraction and is associated with age and steroid treatment duration in duchenne muscular dystrophy. J Am Heart Assoc. 2015;4(4)

26. Duan X, Li J, Zhang Q, Zeng Z, Luo Y, Jiang J, Chen Y. Prognostic value of late gadolinium enhancement in dilated cardiomyopathy patients: a metaanalysis. Clin Radiol. 2015;70(9):999-1008.

27. Hoit BD. Left atrial size and function: role in prognosis. J Am Coll Cardiol. 2014;63(6):493-505.

28. Kemp CD, Conte JV. The pathophysiology of heart failure. Cardiovascular pathology : the official journal of the Society for Cardiovascular Pathology. 2012;21(5):365-71.

29. Brecht A, Oertelt-Prigione S, Seeland U, Rucke M, Hattasch R, Wagelohner T, Regitz-Zagrosek V, Baumann G, Knebel F, Stangl V. Left atrial function in preclinical diastolic dysfunction: two-dimensional speckle-tracking echocardiography-derived results from the BEFRI trial. Journal of the American Society of Echocardiography : official publication of the American Society of Echocardiography. 2016;

30. Parent JJ, Towbin JA, Jefferies JL. Medical therapy leads to favorable remodeling in left ventricular non-compaction cardiomyopathy: dilated phenotype. Pediatr Cardiol. 2016:37(4):674-7.

31. Cheng H, Zhao S, Jiang S, Lu M, Yan C, Ling J, Zhang Y, Liu Q, Ma N, Yin G, et al. Comparison of cardiac magnetic resonance imaging features of isolated left ventricular non-compaction in adults versus dilated cardiomyopathy in adults. Clin Radiol. 2011;66(9):853-60.

32. Lieb W, Gona P, Larson MG, Aragam J, Zile MR, Cheng S, Benjamin EJ, Vasan RS. The natural history of left ventricular geometry in the community: clinical correlates and prognostic significance of change in LV geometric pattern. JACC Cardiovascular imaging. 2014;7(9):870-8.

\section{Submit your next manuscript to BioMed Central and we will help you at every step:}

- We accept pre-submission inquiries

- Our selector tool helps you to find the most relevant journal

- We provide round the clock customer support

- Convenient online submission

- Thorough peer review

- Inclusion in PubMed and all major indexing services

- Maximum visibility for your research

Submit your manuscript at www.biomedcentral.com/submit 